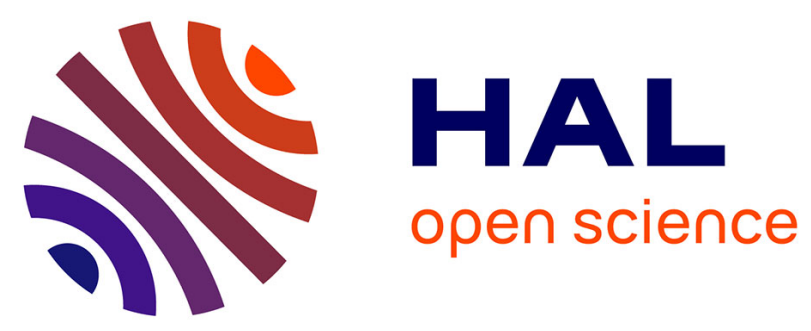

\title{
Is structural sensitivity a problem of oversimplified biological models? Insights from nested Dynamic Energy Budget models.
}

Clement Aldebert, Bob W Kooi, David Nerini, Jean-Christophe Poggiale

\section{To cite this version:}

Clement Aldebert, Bob W Kooi, David Nerini, Jean-Christophe Poggiale. Is structural sensitivity a problem of oversimplified biological models? Insights from nested Dynamic Energy Budget models. . Journal of Theoretical Biology, In press, 10.1016/j.jtbi.2018.03.019 . hal-01742401

\section{HAL Id: hal-01742401 \\ https://hal-amu.archives-ouvertes.fr/hal-01742401}

Submitted on 24 Mar 2018

HAL is a multi-disciplinary open access archive for the deposit and dissemination of scientific research documents, whether they are published or not. The documents may come from teaching and research institutions in France or abroad, or from public or private research centers.
L'archive ouverte pluridisciplinaire HAL, est destinée au dépôt et à la diffusion de documents scientifiques de niveau recherche, publiés ou non, émanant des établissements d'enseignement et de recherche français ou étrangers, des laboratoires publics ou privés. 


\section{Is structural sensitivity a problem of oversimplified}

2 biological models? Insights from nested Dynamic

\section{s Energy Budget models.}

${ }_{4}$ Clement Aldebert $^{\mathrm{ab}, *}$, Bob W Kooi ${ }^{\mathrm{c}}$, David Nerini ${ }^{\mathrm{a}}$, Jean-Christophe Poggiale ${ }^{\mathrm{a}}$

5 a Mediterranean Institute of Oceanography, Aix-Marseille University, Toulon University,

- CNRS/INSU, IRD, MIO, UM 110, 13288, Marseille, Cedex 09, France.

7 b University of Zurich, Institute of Evolutionary Biology and Environmental Studies,

\& Winterthurerstrasse 190, 8057 Zurich, Switzerland

9 c Faculty of Science, VU University, de Boelelaan 1085, 1081 HV Amsterdam, The

10 Netherlands

$11 *$ corresponding author

Keywords model sensitivity ; functional response ; metabolism ; bifurcations ; chemostat

\section{Highlights}

- Four predator-prey models in chemostat are built from Dynamic Energy Budget theory

- Structural sensitivity to functional response formulation is investigated

- The less detailed metabolic model (Monod) leads to higher structural sensitivity

- Structural sensitivity is lower in mass-balanced models including maintenance

Authorship CA performed the research. All authors contributed to the design and discussion. CA wrote the paper and all authors contributed to revisions.

\section{Conflicts of interest none.}




\section{${ }_{23}$ Abstract}

Many current issues in ecology require predictions made by mathematical models, which are built on somewhat arbitrary choices. Their consequences are quantified by sensitivity analysis to quantify how changes in model parameters propagate into an uncertainty in model predictions. An extension called structural sensitivity analysis deals with changes in the mathematical description of complex processes like predation. Such processes are described at the population scale by a specific mathematical function that is taken among similar ones, a choice that can strongly drive model predictions. However, it has only been studied in simple theoretical models. Here, we ask whether structural sensitivity is a problem of oversimplified models. We found in predator-prey models describing chemostat experiments that these models are less structurally sensitive to the choice of a specific functional response if they include mass balance resource dynamics and individual maintenance. Neglecting these processes in an ecological model (for instance by using the well-known logistic growth equation) is not only an inappropriate description of the ecological system, but also a source of more uncertain predictions. 


\section{Introduction}

Facing current socio-environmental issues, such as species extinctions and loss of ecosystem services, requires to make ecological predictions with a level of accuracy that is not yet achieved (Mouquet et al., 2015; Morozov, 2017; Pennekamp et al., 2017). Uncertainty arises in predictions made by mathematical models, which are perceived as objective tools but remain simplified representations built on somewhat arbitrary choices Anderson, 2005 , 2010). Among these choices, two types can be distinguished. The first ones are the processes and components (e.g. species, nutrients) to include, which are often a consensus between scientists from different ecology-related disciplines (animal and plant biology, microbiology, chemistry, physics, Demongeot et al., 2009). Those choices are assumptions that can be discussed, and testing their consequences on predictions helps to improve ecological theories. The second type of choices is still an open issue and is the mathematical function selected to model a given process (Lafferty et al. 2015). A process can be described by many functions that fit available data with the same accuracy but that are based on different assumptions. Whereas different assumptions about the emerging process shape (e.g. density-dependence, group behaviour) have been widely considered in the literature, the choice between similar functions (i.e mechanisms) to model the same process shape (e.g. process rate increases with population abundance) has received only little attention in ecology. However, this attention has increased in the past decade since the preliminary work by Myerscough et al. (1996) and Wood \& Thomas (1999), followed by Gross et al. (2004) and Fussmann \& Blasius (2005). Recent studies indicate that this choice can deeply affect both qualitative and quantitative predictions (Cordoleani et al., 2011), including those at the food web level or about system resilience (Aldebert et al., 2016a b). This concept extends the idea of parameter sensitivity, as it becomes the sensitivity of model predictions to any change in parameter values and/or model formulation (see Cordoleani et al., 2011, for a proper mathematical formulation). This more general concept has been coined structural sensitivity.

Structural sensitivity emerges if several scales of organization, space and/or time are 
entangled in a complex process. Such a process is for instance predation. Predation involves individual to population level mechanisms that are often summarized at the population scale by one function, the functional response (Solomon, 1949). Many functional responses can be derived depending on the mechanisms considered (Jeschke et al., 2002). A mechanism underlying a formulation (e.g. prey handling) might be relevant, but additional assumptions that translate it into mathematics (e.g. space homogeneity, no individual variability) are almost always violated. Thus, the best formulation from a theoretical point of view might not be the best quantitative description of data. As this uncertainty propagates into model predictions, Gross \& Feudel (2006) and Adamson \& Morozov (2012) proposed approaches based on generic (partially-specified) models. These generalized models avoid the issue of structural sensitivity and are useful to draw widely applicable conclusions in theoretical studies (Gross et al. 2009). However, such models hardly consider non-equilibrium dynamics (Kuehn \& Gross, 2013) and tell nothing about the existence of alternative stable states, two important characteristics of living systems (Fussmann et al., 2000; Scheffer et al., 2012) that can be affected by structural sensitivity (Aldebert et al., 2016b).

In this study, we suggest an alternative way to deal with structural sensitivity that applies to systems with non-equilibrium dynamics and alternative stable states. Structural sensitivity has only been studied in theoretical population models, where population growth is logistic for the prey and proportional to the feeding rate for the predator. So, one may think that structural sensitivity is a problem of oversimplified models. We test this hypothesis by presenting the first study on structural sensitivity in ecosystem models (sensu with explicit resource dynamics) that include various level of details to describe individual metabolism.

Modelling individual metabolism requires to add processes and create model sensitivity to their formulation. Mechanistic formulations of metabolic processes can be derived from Dynamic Energy Budget (DEB) theory (Kooijman, 2010; Jusup et al., 2017). This reductionist theory focuses on the individual level, as it allows to make easy mass and energy budgets. As a consequence, the formulation of metabolic processes is constrained by the 
laws of thermodynamics. Another advantage of DEB theory is that it provides a level of abstraction that allows generalization to many living organisms.

To test whether structural sensitivity is a result of model oversimplification, we focus on a predator-prey system of dividing unicellular organisms living in a chemostat-like environment (figure 1a). This system is modelled using different functional responses (figure 1b) and levels of metabolic details (figure 1 1F). For the metabolism, we consider a predator-prey model based on DEB theory (Kooi \& Kooijman, 1994b) that describes chemostat experiments and includes two buffers between feeding and population growth: an energy reserve and maintenance costs. These two features are neglected at limit cases of this model, leading to three simpler models: Droop (1973), Marr-Pirt (Marr et al., 1963) and Monod (1942) models. Thus, these four models are nested within the framework of DEB theory.

Next section presents the nested predator-prey models. Then, their predictions are analyzed and discussed in the light of the general question: Is structural sensitivity a problem of the oversimplified biological models? Discussion ends with a synthesis of research on structural sensitivity that leads to a guidance for ecologists in their modelling choices.

\section{Models}

\subsection{Functional responses to model predation}

To model predation, we consider the next three functions (figure 1 $1 \mathrm{~b}$ ):

$$
F^{H}(X)=\frac{j_{X A m}^{H} X}{X+K^{H}}, F^{I}(X)=j_{X A m}^{I}\left(1-\exp \left(-X / K^{I}\right)\right), F^{t}(X)=j_{X A m}^{t} \tanh \left(X / K^{t}\right),
$$

where $X$ is prey biomass, $j_{X A m}$ is the maximum assimilation rate and $j_{X A m} / K$ is the function slope at 0 . The classical Holling functional response $F^{H}$ assumes that a predator splits its activity between searching and handling prey (Holling, 1965). It is equivalent to

Michaëlis-Menten function for enzyme kinetics. Ivlev functional response $F^{I}$ is based on 


\section{a. Experimental device}

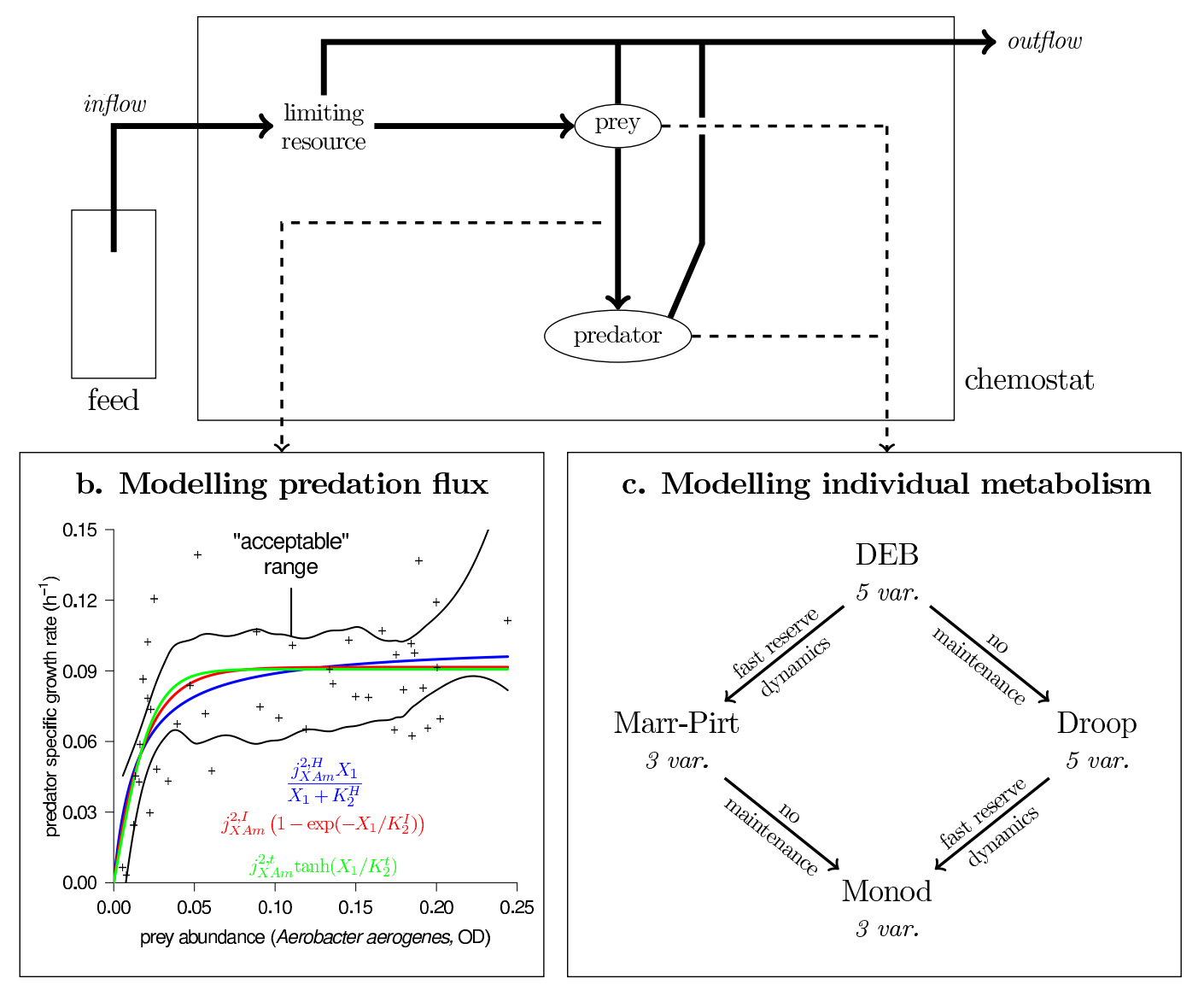

Figure 1. Sketch of the study. We compare predictions of predator-prey system in a chemostat-like environment (a) modelled with one of three mathematical functions (color, "acceptable range" is the $95 \%$ confidence interval of a non-parametric kernel regression) to model predation (b) and one of four nested models to model individual metabolism (c). 
digestion (Ivlev, 1955). Conversely, the hyperbolic tangent function $F^{t}$ has no theoretical basis, but it happens to be an appropriate description of data (Jassby \& Platt, 1976) and it is used in some population models (Fussmann \& Blasius, 2005; Cordoleani et al., 2011). The three prey-dependent functions (1) are type-II functional responses (they vanish at zero, are strictly increasing, concave and saturating). However, the same exercise can be performed with more complex functions like type-III (sigmoid) or ratio-dependent functional responses.

\subsection{Predator-prey models}

Starting from a DEB model for unicellular dividing individuals (Appendix A), Kooi \& Kooijman (1994b) proposed the following model (referred as DEB model) to describe a predatorprey system living an environment described by a chemostat:

$$
\left\{\begin{aligned}
\frac{d e_{1}}{d t} & =\dot{k}_{E}^{1}\left(f_{1}\left(X_{0}\right)-e_{1}\right) \\
\frac{d e_{2}}{d t} & =\dot{k}_{E}^{2}\left(f_{2}\left(X_{1}\right)-e_{2}\right) \\
\frac{d X_{0}}{d t} & =\dot{h}\left(X_{r}-X_{0}\right)-F_{1}\left(X_{0}\right) X_{1} \\
\frac{d X_{1}}{d t} & =\left(\frac{\dot{k}_{E}^{1} e_{1}-\dot{k}_{M}^{1} g_{1}}{e_{1}+g_{1}}-\dot{h}\right) X_{1}-F_{2}\left(X_{1}\right) X_{2} \\
\frac{d X_{2}}{d t} & =\left(\frac{\dot{k}_{E}^{2} e_{2}-\dot{k}_{M}^{2} g_{2}}{e_{2}+g_{2}}-\dot{h}\right) X_{2} .
\end{aligned}\right.
$$

The prey (structure $X_{1}$ and scaled reserve density $e_{1}$ ) feeds on an inorganic resource (concentration $X_{0}$ ) and is eaten by a predator (structure $X_{2}$ and scaled reserve density $e_{2}$ ), with $f_{i}\left(X_{i-1}\right):=F_{i}\left(X_{i-1}\right) / j_{X A m}^{i}, i=1,2$ being scaled functional responses. The biological parameters $\dot{k}_{E}^{i}\left(\right.$ in $\left.^{-1}\right), \dot{k}_{M}^{i}$ (in $\mathrm{h}^{-1}$ ) and $g_{i}$ (no unit) are the specific energy conductance, somatic maintenance rate and energy investment rate of species $i$ respectively. Two environmental parameters describe the chemostat, its dilution rate $\dot{h}\left(\mathrm{in}^{-1}\right)$ and the resource 
concentration in the feed $X_{r}$. This model assumes that the predator digests only prey structure, as adding reserve digestion does not improve the fit to data (Kooijman, 2010, p357).

Marr-Pirt model is a specific case of the DEB model (2) where reserve dynamics is assumed to be infinitely fast $\left(\dot{k}_{E}^{i} \rightarrow+\infty\right)$. It implies that the scaled reserve density is a function of the available food $\left(e_{i}(t)=f_{i}\left(X_{i-1}(t)\right)\right)$ at the same time. Taking also $g_{i} \rightarrow+\infty$, the growth rate of structure becomes $\dot{\mu}_{i} f_{i}\left(X_{i-1}\right)-\dot{k}_{M}^{i}$, where $\dot{\mu}_{i}=\dot{k}_{E}^{i} / g_{i}$. The new parameter $\dot{\mu}_{i}\left(\right.$ in $\left.^{-1}\right)$ is the maximum growth rate of the population. Then, the DEB model (2) simplifies into the following three-dimensional system based on Marr-Pirt model:

$$
\left\{\begin{array}{l}
\frac{d X_{0}}{d t}=\dot{h}\left(X_{r}-X_{0}\right)-f_{1}\left(X_{0}\right) j_{X A m}^{1} X_{1} \\
\frac{d X_{1}}{d t}=\left(\dot{\mu}_{1} f_{1}\left(X_{0}\right)-\dot{k}_{M}^{1}-\dot{h}\right) X_{1}-f_{2}\left(X_{1}\right) j_{X A m}^{2} X_{2} \\
\frac{d X_{2}}{d t}=\left(\dot{\mu}_{2} f_{2}\left(X_{1}\right)-\dot{k}_{M}^{2}-\dot{h}\right) X_{2}
\end{array}\right.
$$

Droop and Monod models are specific cases of the DEB and Marr-Pirt models respectively, where $\dot{k}_{M}^{1}=\dot{k}_{M}^{2}=0$, i.e. maintenance costs are neglected for both species.

\subsection{Analysis of model predictions}

To get a global picture of model predictions, we focus on the type of predicted asymptotic dynamics (the state that the system will reach after a sufficient amount of time) like species survival and equilibrium situation vs. predator-prey oscillations. A qualitative change between asymptotic dynamics, like a species extinction or the onset of predator-prey oscillations, occurs at a threshold on parameter values called bifurcation (Kuznetsov, 2004). Bifurcations that correspond to a sudden collapse or transition to an alternative state of the system are also known as tipping point and critical transition in the ecological literature (Scheffer et al., 2012). We provide some analytical results on bifurcations related to species extinction in Appendix B. The next section presents the full results with all the 
model bifurcations as thresholds on environmental parameters ( $\dot{h}$ and $X_{r}$ ), computed using numerical methods (Dhooge et al. 2006$)$ for a given functional response and given values of the biological parameters.

Biological parameters are set to numerical values that describe a chemostat experiment by Dent et al. (1976) where Escherichia coli grows on glucose and is eaten by Dictyostelium discoides. This parameter estimation was performed by Kooi \& Kooijman (1994b) for the four chemostat models using Holling-II functional response for each species. As functional response data were not available (Kooi \& Kooijman fitted the predicted model dynamics on temporal data), we mimic the situation in figure 1 $1 \mathrm{~b}$ following Aldebert et al. (2016a) $\mathrm{b}$ ): parameters of $F^{I}(X)$ and $F^{t}(X)$ were set to minimize the Euclidean distance between these functions and the Holling-II functional response (Appendix A), which is equivalent to fit all functions to data if functional response data were available. For Monod model, we also used data from an experiment by Canale et al. (1973) where Aerobacter aerogenes grows on carbohydrates and is eaten by Tetrahymena pyriformis (functional response data were available and used to parameterize the three functional responses, see figure 1 $1 \mathrm{~b})$. The predictions by Monod models are qualitatively the same between both experiments, but for numerical reasons (see next section) they are easier to visualize with parameter values from the experiment by Canale et al. (1973). For each model, only the predator functional response (flux from the prey to the predator) is changed. We kept Holling-II for the prey functional response (flux from the resource to the prey) as changing it has only a little quantitative effect on our results (data not shown). All parameter values are provided in Appendix A.

\section{Results}

Overall, the three functional responses lead to the same qualitative pattern of predicted dynamics (dynamics, type of bifurcations) in each predator-prey model, except for Monod 
model (figures 2, 3, Appendix C). Monod model together with the hyperbolic tangent can predict the coexistence of two alternative stable states (figure 33 ) corresponding to preypredator coexistence either at equilibrium or with oscillations. These alternative states are not predicted with the two other functional responses in Monod model (figure 2 $\mathrm{d}$ ). Note that results for Monod model are qualitatively the same with data from both Dent et al. (1976) and Canale et al. (1973) experiments (Appendix C), and only the latter is presented in figures $2 \mathrm{~d}-3 \mathrm{~d}$ as results are easier to visualize for numerical reasons. In DEB, Droop and Marr-Pirt models, bifurcations occur at slightly different values between functional responses, but only in a limited range of environmental conditions (low resource concentration in the feed) corresponding to $1 \%$ to $\approx 25 \%$ of the bifurcation diagrams (details in Appendix C) and bifurcation diagrams have the same general pattern.

The pattern of predicted dynamics is also affected by the level of metabolic details included in the model. The DEB model can predict with all functional responses the coexistence of alternative stable states (figures $2 \mathrm{a}-3 \mathrm{a}$ ). These alternative dynamics were not reported in the previous analysis of the DEB model (Kooi \& Kooijman, 1994a), and they are not predicted if either maintenance or reserve dynamics are not included. Not including reserve dynamics also increases the range of dilution rates that predict species survival (figures $2 \mathrm{~b}-3 \mathrm{~b}$ ). Conversely, not including maintenance decreases the range of dilution rates that predict species survival, especially for the prey (figures 22-3r). In addition, species are predicted to survive even at infinitely small dilution rates, as they do not have to pay maintenance costs to survive. This last effect is also found if both maintenance and reserve dynamics are not included to model the same species, but species are predicted to survive in a larger range of dilution rates (Appendix $\mathrm{C}$ ). 
explicit

reserve dynamics fast reserve

dynamics a. DEB

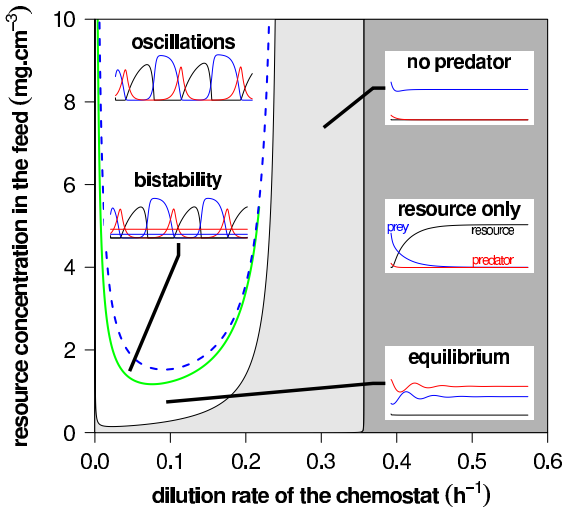

c. Droop

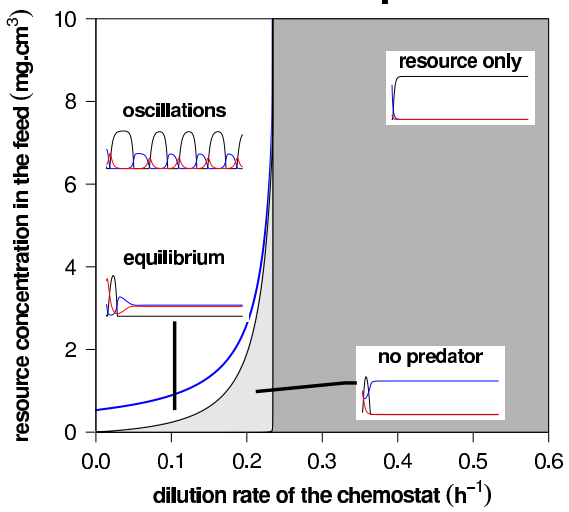

b. Marr-Pirt

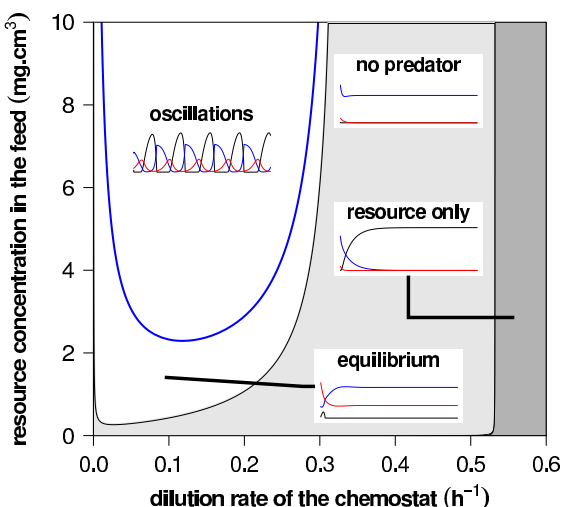

d. Monod

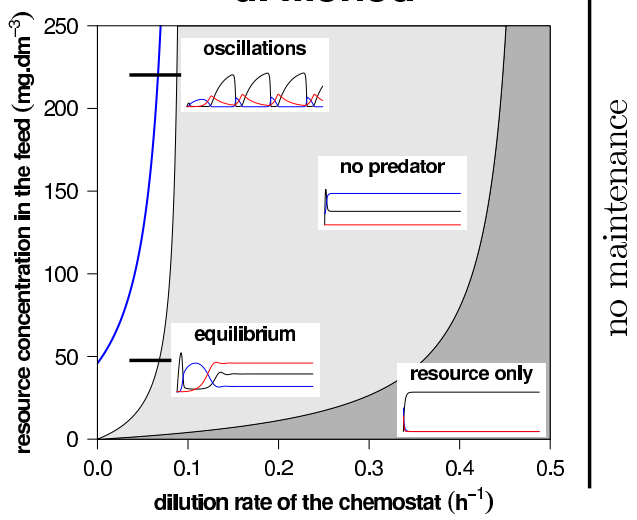

Figure 2. Predictions made with the four models of individual metabolism and Holling-II functional response. Predictions of each model are summarized by a bifurcation diagram which indicates the predicted qualitative dynamics (small panels) as a function of environmental parameters, the thresholds on environmental parameters are bifurcations (bif.) being indicated by the curves (black: transcritical bif. = species extinction, plain/dashed blue: supercritical/subcritical Hopf bif. = stable/unstable oscillations, green: limit point for cycles bif. = both stable and unstable oscillations). Biological parameters represent Dictyostelium discoideum feeding on Escherichia coli in (a-c) and Tetrahymena pyriformis feeding on Aerobacter aerogenes in (d). Qualitative differences between figures $2 \mathrm{~d}$ and $3 \mathrm{~d}$ drawn for the first species are the same but are harder to visualize (Appendix C). Results for Ivlev's functional response are qualitatively the same (Appendix C). 
explicit

fast reserve

reserve dynamics

dynamics

a. DEB

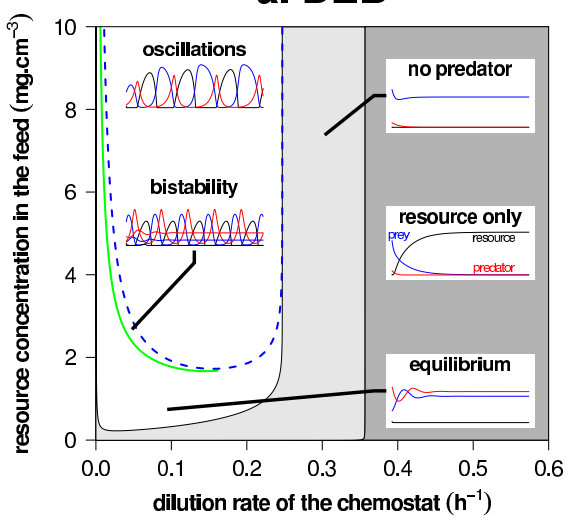

c. Droop

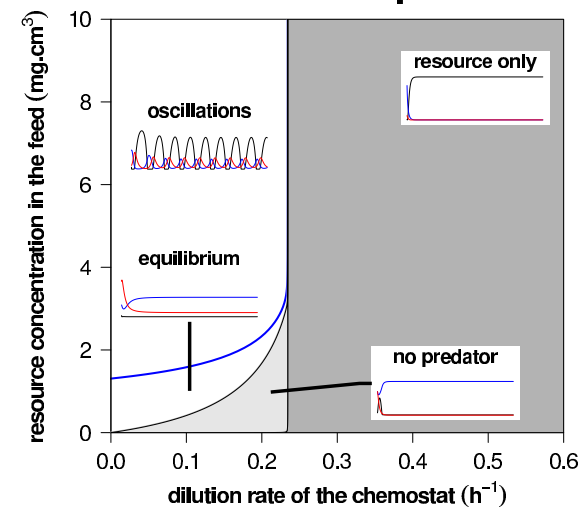

b. Marr-Pirt

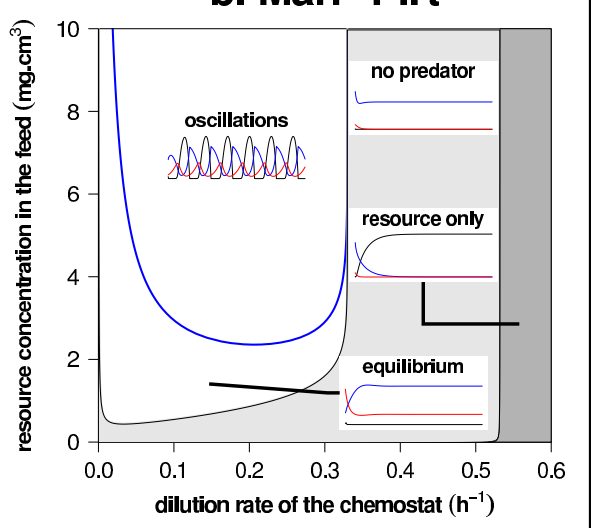

d. Monod

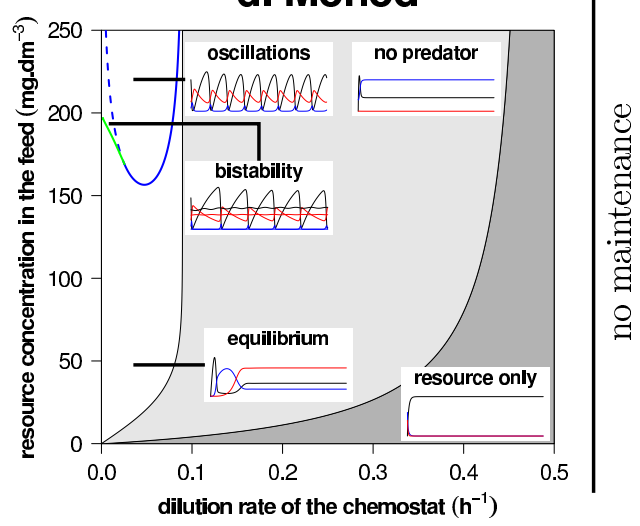

Figure 3. Predictions made with the four models of individual metabolism and an hyperbolic tangent functional response. Legend and modelled species are the same as in figure 2. In (d), there is a Bautin bifurcation point where the Hopf bifurcation (blue) switches from supercritical (plain) to subcritical (dashed) and a limit point for cycles bifurcation curve (green) emanates. 


\section{Discussion}

Including more details on individual metabolism decreases the structural sensitivity of the population model. Indeed, structural sensitivity is higher with Monod model, as functional response formulation affects the qualitative pattern (type of bifurcations and predicted dynamics) of model predictions more than in the three more complex models: Marr-Pirt, Droop and DEB models. Also, in these models, only $1 \%$ to $\approx 25 \%$ of a bounded part of the bifurcation diagrams is affected by the change of functional response. This is significantly lower than the $26 \%$ to $64 \%$ of changes that we found in Bazykin's predator-prey model Aldebert et al., 2016b) where changes occur in a non-bounded subspace of parameter values. Here, the three less sensitive models include at least maintenance or reserve dynamics.

Putting the previous conclusion into a broader framework of predator-prey models, an additional requirement to avoid a strong structural sensitivity (i.e. changes in the type of bifurcations and dynamics) is to include an explicit description of resource dynamics (figure 4 ). Explicit resource dynamics are used in mass-balance models (here chemostat), whereas other models use a logistic growth equation for the prey. The logistic growth equation is phenomenological, so its use may be less justified in comparison to a mechanistic mass-balance equation of resource uptake (that might be a chemostat or not). These two approaches can lead to very different dynamics in food chain models (Kooi et al., 1998). In addition to these limits of the logistic growth equation, we found that its use makes models more sensitive to changes in the mathematical representation of trophic interactions. Here, we will not discuss which model features (maintenance, reserve dynamics) make a model more or less sensitive to predator functional response in terms of bifurcations location. Indeed, these quantitative changes will depend on the biological parameter values that correspond here to two specific experiments. Also, a deeper quantitative analysis including the predicted population size (as in Cordoleani et al. 2011, figure 5) across the whole bifurcation diagrams would require a too high computational effort with two environmental parameters that vary and non-equilibrium dynamics. In addition, existing metrics (Cordoleani et al., 2011; Adamson \& Morozov, 2012) 


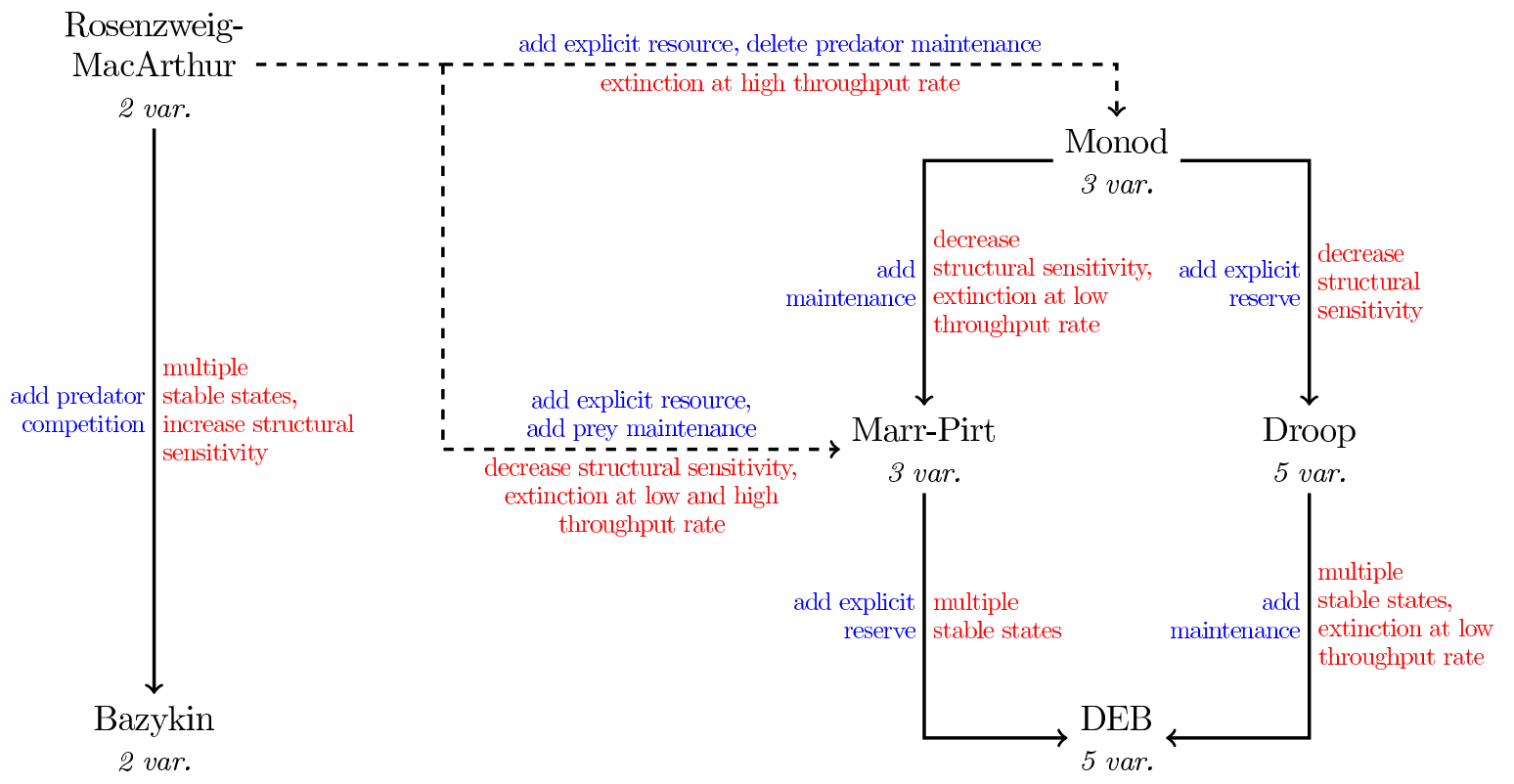

Figure 4. Changes in model assumptions (blue) and their consequences on model predictions (red). Structural sensitivity is here quantified in terms of qualitative change in bifurcations type. For Rosenzweig \& MacArthur (1963) and Bazykin (1998) models, structural sensitivity analysis is presented in Fussmann \& Blasius (2005) and Aldebert et al. (2016b) respectively. Arrows indicate either that the starting model is a limit case (plain) or an approximation (dashed) of the ending model. Details in Appendix D.

were designed to compare models with one stable and need to be first extend to compare models that might predict a different number of stable states (e.g. bistability area in figure $3 \mathrm{~d}$ that does not exist in figure $2 \mathrm{~d}$, or in figure $2 \mathrm{a}$ vs. figure $2 \mathrm{~b}$ ).

The parameter values of Ivlev and the hyperbolic tangent functional responses are only estimated by fitting them to Holling-II functional response. The latter is not parameterized from functional response data, but by optimizing all parameter values so that model dynamics fit empirical data (Kooi \& Kooijman, 1994b). Using this fitting procedure to estimate all parameters for each functional response would take into account the co-variation of some parameters (Lika et al. 2011). Thus, a possible way to deal with structural sensitivity is to acquire data on both processes (here the functional response) and temporal dynamics of the system. Doing this would include the predicted location of thresholds like species extinction and the onset of predator-prey oscillations as constraints in functional response estimation. 
Apart from the synergistic effect of maintenance and reserve on the coexistence of alternative stable states, reserve has a smaller impact on model predictions than maintenance. Maintenance implies that a species disappears if resource input (here the dilution rate) is not high enough to overcome its cost. This result was already known for models without reserve (Nisbet et al., 1983; Kooi, 2003), and we extend it here to models with explicit reserve. Note that the four models predict different thresholds of environmental parameters for species invasion. Indeed, biological parameter values are optimized from one data set corresponding to a single environmental condition. Thus, extrapolations from this reference condition are likely to vary between models. From a biological point of view, maintenance (linear mortality in community models) is a basic process that should be considered. In addition, using explicit reserve without maintenance costs (Droop model) lead to the worst fit to data (Kooi \& Kooijman, 1994b). So, despite including reserve is relevant for many reasons (Kooijman, 2010, section 1.1.3), it seems to be less important than maintenance.

When multiple resources limit the growth of the prey, the way their co-limited uptake is modelled deeply affects the predicted dynamics of a predator-prey system (Poggiale et al. 2010). These dynamics also change if maintenance is explicitly taken into account or not. The uptake of multiple resources can be modeled in a mechanistic way through the concept of Synthesising Units (Kooijman, 2010, chapter 3) that describe enzymatic pathways. Here, we found that the predation formulation for the prey (i.e. resource uptake) has little effects on model dynamics with one limiting resource. With multiple resources, model sensitivity to the formulation of both predation and co-limited uptake remains to be assessed.

In addition to the number of limiting resources, the number of species in interaction also influences system dynamics and structural sensitivity (Aldebert et al., 2016a). Here, we considered two species in interaction, but simple three-species food webs can have more complicated dynamics with Monod and Marr-Pirt models (Kooi \& Boer, 2001), and the situation might be more entangled for the DEB model (2). Moreover, the sensitivity functional response formulation would be more complex to analyze for these models. 


\section{Conclusion}

As a conclusion, the answer to the general question: Is structural sensitivity problem of oversimplified biological models? is Yes in the context of our study. Here we found a lower structural sensitivity in predator-prey models that include explicit resource dynamics and maintenance (or reserve dynamics, figure 4). Thus, including these processes allows to achieve three goals: (i) description of relevant processes, (ii) a better fit to available data (Kooi \& Kooijman, 1994b; Poggiale et al., 2010), and (iii) more accurate predictions (forecast of unknown situations) with respect to uncertainty in the mathematical formulation of complex processes like predation. For this last point, one avoids the worst effect of structural sensitivity (qualitative change in predictions). However, some uncertainty in model predictions (quantitative predictions, precise bifurcation values) remain, which motivate ongoing researches on the quantification of structural sensitivity to allow the communication of model predictions together with their uncertainty. Finally, including explicit resource dynamics and maintenance only slightly increases model complexity, which allows to keep models tractable. Thus, including these processes is a promising way to deal with structural sensitivity, including in systems with non-equilibrium dynamics and alternative stable states. So, the extension of our results to operational models based on multiple species and resources is an open way of research. Another open way is to check that our guidance on the use of predator-prey models holds for parameter values that describe data for a wide range of species, which would be a critical advance toward more accurate predictions in ecology.

\section{Acknowledgements}

We thanks SALM Kooijman and the participants of the 8th and 9th International Course and Symposia on DEB theory (Marseilles, France, 2015 and Tromsø, Norway, 2017). We acknowledge Mathias Gauduchon, Owen Petchey and Aurelie Garnier for discussion. The PhD scholarship of CA was funded by the French ministry of Higher Education and Research. 
CA, DN and JCP received funding from European FEDER Fund under project 1166-39417.

\section{References}

Adamson, MW, Morozov, AY. 2012. When can we trust our model predictions? Unearthing structural sensitivity in biological systems. Proceedings of the Royal Society of London A, $469(2149)$.

Aldebert, C, Nerini, D, Gauduchon, M, Poggiale, JC. 2016a. Does structural sensitivity alter complexity-stability relationships? Ecological Complexity, 28, 104-112.

Aldebert, C, Nerini, D, Gauduchon, M, Poggiale, JC. 2016b. Structural sensitivity and resilience in a predator-prey model with density-dependent mortality. Ecological Complexity, 28, 163-173.

Anderson, TR. 2005. Plankton functional type modelling: running before we can walk? Journal of Plankton Research, 27(11), 1073-1081.

Anderson, TR. 2010. Progress in marine ecosystem modelling and the "unreasonable effectiveness of mathematics". Journal of Marine Systems, 81, 4-11.

Bazykin, AD. 1998. Nonlinear dynamics of interacting populations. World Scientific, Singapore. $193 \mathrm{pp}$.

Canale, RP, Lustig, TD, Kehrberger, PM, Salo, JE. 1973. Experimental and mathematical modeling studies of protozoan predation on bacteria. Biotechnology and Bioengineering, 15, 707-728.

Cordoleani, F, Nerini, D, Gauduchon, M, Morozov, A, Poggiale, JC. 2011. Structural sensitivity of biological models revisited. Journal of Theoretical Biology, 283, 82-91.

Demongeot, J, Françoise, JP, Nerini, D. 2009. From biological and clinical experiments to mathematical models. Phil. Trans. R. Soc. A, 367, 4657-4653. 
Dent, VE, Bazin, MJ, Saunders, PT. 1976. Behaviour of Dictyostelium discoideum Amoebae and Escherichia coli grown together in chemostat culture. Archives in Microbiology, 109, $187-194$.

Dhooge, A, Govaerts, W, Kuznetsov, YuA, Mestrom, W, Riet, AM, Sautois, B. 2006. MATCONT and CL MATCONT: Continuation toolboxes in matlab. http://matcont.ugent. $\mathrm{be} /$.

Droop, MR. 1973. Some thoughs on nutrient limitation in algae. Journal of Phycology, 9, $264-272$.

Fussmann, GF, Blasius, B. 2005. Community response to enrichment is highly sensitive to model structure. Biology Letters, 1(1), 9-12.

Fussmann, GF, Ellner, SP, Shertzer, KW, Hairston Jr, NG. 2000. Crossing the Hopf bifurcation in a live predator-prey system. Science, 290, 1358-1360.

Gross, T, Feudel, U. 2006. Generalized models as a universal approach to the analysis of nonlinear dynamical systems. Physical Review E, 73(1), 016205.

Gross, T, Ebenhöh, W, Feudel, U. 2004. Enrichment and foodchain stability: the impact of different forms of predator-prey interaction. Journal of Theoretical Biology, 227, 349-358.

Gross, T, Rudolf, L, Levin, SA, Dieckmann, U. 2009. Generalized models reveal stabilizing factors in food webs. Science, 325, 747-750.

Holling, CS. 1965. The functional response of predators to prey density and its role in mimicry and population regulation. Memoirs of the Entomological Society of Canada, 45, 3-60.

Ivlev, VS. 1955. Experimental ecology of the feeding of fishes. Pischepromizdat, Moscow. 302 pp. (translated from Russian by D. Scott, Yale University Press, New Haven, 1961). 
Jassby, AD, Platt, T. 1976. Mathematical formulation of the relationship between photosynthesis and light for phytoplankton. Limnology and Oceanography, 21(4), 540-547.

Jeschke, JM, Kopp, M, Tollrian, R. 2002. Predator functional response: discriminating between handling and digesting prey. Ecological Monographs, 72(1), 95-112.

Jusup, M, Sousa, T, Domingos, T, Labinac, V, Marn, N, Wang, Z, Klanjišček, T. 2017. Physics of metabolic organization. Physics of Life Reviews, 20, 1-39.

Kooi, BW. 2003. Numerical bifurcation analysis of ecosystems in a spatially homogeneous environment. Acta biotheoretica, 51(3), 189-222.

Kooi, BW, Boer, MP. 2001. Bifurcations in ecosystem models and their biological interpretation. Applicable Analysis, 77, 29-59.

Kooi, BW, Kooijman, SALM. 1994a. Existence and stability of microbial prey-predator systems. Journal of Theoretical Biology, 170, 75-85.

Kooi, BW, Kooijman, SALM. 1994b. The transient behaviour of food chains in chemostat. Journal of Theoretical Biology, 170, 87-94.

Kooi, BW, Boer, MP, Kooijman, SALM. 1998. On the use of the logistic equation in models of food chains. Bulletin of Mathematical Biology, 60, 231-246.

Kooijman, SALM. 2010. Dynamic energy budget theory for metabolic organisation. 3rd edn. Cambridge University Press, Cambridge. 514 pp.

Kuehn, C, Gross, T. 2013. Nonlocal generalized models of predator-prey systems. Discrete and Continuous Dynamical Systems B, 18(3), 693-720.

Kuznetsov, YuA. 2004. Elements of applied bifurcation theory. 3rd edn. Springer, New York. $361 \mathrm{pp}$. 
Lafferty, KD, DeLeo, G, Briggs, CJ, Dobson, AP, Kuris, AM. 2015. A general consumerresource population model. Science, 349, 854-857. (doi:10.1126/science.aaa6224).

Lika, K, Kearney, MR, Freitas, V, van der Veer, HW, van der Meer, J, Wijsman, JWM, Pecquerie, L, Kooijman, SALM. 2011. The "covariation method" for estimating the parameters of the standard Dynamic Energy Budget model I: Philosophy and approach. Journal of Sea Research, 66, 270-277.

Marr, AG, Nilson, EH, Clark, DJ. 1963. The maintenance requirement of Escherichia coli. Annals New York Academy of Sciences, 102, 536-548.

Monod, J. 1942. Recherches sur la croissance des cultures bactériennes (Researches on the growth of bacterial cultures, in French). 2nd edn. Hermann, Paris. 459 pp.

Morozov, A. 2017. Introduction to the themed issue: Uncertainty, sensitivity and predictability in ecology: Mathematical challenges and ecological applications. Ecological Complexity, 32, 131-133.

Mouquet, N, Lagadeuc, Y, Devictor, V, Doyen, L, Duputie, A, Eveillard, D, Faure, D, Garnier, E, Gimenez, O, Huneman, P, et al. 2015. Predictive ecology in a changing world. Journal of Applied Ecology, 52, 1293-1310.

Myerscough, MR, Darwen, MJ, Hogarth, WL. 1996. Stability, persistence and structural stability in a classical predator-prey model. Ecological Modelling, 89, 31-42.

Nisbet, RM, Cunningham, A, Gurney, WSC. 1983. Endogenous metabolism and the stability of microbial prey-predator systems. Biotechnology and Bioengineering, 25, 301-306.

Pennekamp, F, Adamson, MW, Petchey, OL, Poggiale, JC, Aguiar, M, Kooi, BW, Botkin, DB, DeAngelis, DL. 2017. The practice of prediction: What can ecologists learn from applied, ecology-related fields? Ecological Complexity, 32, 156-167. 
Poggiale, JC, Baklouti, M, Queguiner, B, Kooijman, SALM. 2010. How far details are important in ecosystem modelling: the case of multi-limiting nutrients in phytoplanktonzooplankton interactions. Philosophical Transactions of the Royal Society of London B, 365, 3495-3507.

Rosenzweig, ML, MacArthur, RH. 1963. Graphical representation and stability conditions of predator-prey interaction. The American Naturalist, 97(895), 209-223.

Scheffer, M, Carpenter, SR, Lenton, TM, Bascompte, J, Brock, WA, Dakos, V, van de Koppel, J, van de Leemput, IA, Levin, SA, van Nes, EH, Pascual, M, Vandermeer, J. 2012. Anticipating critical transitions. Science, 338, 344-348.

Solomon, ME. 1949. The natural control of animal populations. Journal of Animal Ecology, 18(1), 1-35.

Wood, SN, Thomas, MB. 1999. Super-sensitivity to structure in biological models. Proceedings of the Royal Society of London B, 266, 565-570. 